\title{
MCL-1 and BCL-xL-dependent resistance to the BCL-2 inhibitor ABT-199 can be overcome by preventing PI3K/ AKT/mTOR activation in lymphoid malignancies
}

\author{
GS Choudhary ${ }^{1,2}$, S Al-harbi ${ }^{1}$, S Mazumder ${ }^{1}$, BT Hill ${ }^{3}$, MR Smith ${ }^{3}$, J Bodo ${ }^{4}$, ED Hsi' ${ }^{4}$ and A Almasan ${ }^{\star, 1}$
}

Overexpression of anti-apoptotic BCL-2 family members is a hallmark of many lymphoid malignancies, including chronic lymphocytic leukemia (CLL) and non-Hodgkin lymphoma (NHL) that can be targeted with small molecule inhibitors. ABT-199 is a rationally designed BCL-2 homology (BH)-3 mimetic that specifically binds to BCL-2, but not to MCL-1 and BCL-xL. Although the thrombocytopenia that occurs with navitoclax treatment has not been a problem with ABT-199, clinical trials in CLL could benefit by lowering the ABT-199 concentration through targeting other survival pathways. In this study, we investigated the mechanisms of resistance that develops to ABT-199 therapy by generating ABT-199-resistant (ABT199-R) cell lines via chronic exposure of NHL cell lines to ABT-199. Acquired resistance resulted in substantial AKT activation and upregulation of MCL-1 and BCL-xL levels that sequestered BIM. ABT199-R cells exhibited increased MCL-1 stability and failed to activate BAX in response to ABT-199. The ABT-199 acquired and inherent resistant cells were sensitized to treatment with ABT-199 by inhibitors of the PI3K, AKT, and mTOR pathways, NVP-BEZ235 and GS-1101. NVP-BEZ235, a dual inhibitor of p-AKT and $m$ TOR, reduced MCL-1 levels causing BIM release from MCL-1 and BCL-XL, thus leading to cell death by BAX activation. The PI3K $\delta$ inhibitor GS-1101 (idelalisib) downregulated MCL-1 and sensitized ABT199-R cells through AKT-mediated BAX activation. A genetic approach, through siRNA-mediated downregulation of $A K T, M C L-1$, and $B C L-x L$, significantly decreased cell survival, demonstrating the importance of these cell survival factors for ABT-199 resistance. Our findings suggest a novel mechanism that modulates the expression and activity of pro-survival proteins to confer treatment resistance that could be exploited by a rational combination therapeutic regimen that could be effective for treating lymphoid malignancies.

Cell Death and Disease (2015) 6, e1593; doi:10.1038/cddis.2014.525; published online 15 January 2015

Diffuse large B-cell lymphoma (DLBCL), the most common subtype of non-Hodgkin lymphoma is categorized as germinal center B-cell-like and activated B-cell-like disease. ${ }^{1}$ Several gene expression-profiling studies have shown distinct molecular signatures in germinal center and activated B-cell-like disease that differentiate them based on oncogenic dependency and clinical outcome of the disease. ${ }^{2,3} \mathrm{~A}$ hallmark pathway that drives DLBCL tumor progression is mutation in immunoglobulin heavy variable gene rearrangement, causing activation of the B-cell receptor pathway that increases expression of specific receptors that facilitate activation of critical pathways involved in tumor progression and upregulation of anti-apoptotic BCL-2 family proteins, thereby causing chemoresistance and aggressive relapse in the clinic. ${ }^{4-7}$

The role of constitutive PI3K signaling in B cells, particularly of the PI3K $\delta$ isoform that is primarily expressed in hematopoietic cells, has been implicated as a central mechanism for relaying cell survival, adhesion, and proliferative signals. $\mathrm{PI}$ KK $\delta$ via AKT achieves transcriptional, translational, and posttranslational regulation of $\mathrm{BCL}-2$ family proteins by regulating mTOR, GSK3, FOXO, and NF- $k B$. ${ }^{5,8}$ The BCL-2 family members are key regulators of the intrinsic apoptotic pathway and are classified into three classes of proteins based on their structural similarity and function: the anti-apoptotic proteins (BCL-2, BCL-xL, MCL-1, BFL-1, and BCL-w) sequester the BH3-only proteins (BIM, BID, PUMA, and NOXA) that in turn activate the pro-apoptotic proteins (BAX and BAK). In some cases, anti-apoptotic BCL-2 proteins can also sequester pro-apoptotic proteins. BAX/BAK oligomerization causes mitochondrial outer membrane permeabilization, resulting in cytochrome $c$ release and apoptosis. ${ }^{9,10}$ Chronic lymphocytic leukemia (CLL) cells rely on increased expression of antiapoptotic BCL-2 proteins; strategies to restore apoptosis by antagonizing them have led to development of $\mathrm{BH} 3$ mimetics as therapeutic agents that have a robust clinical response with reduced toxicity. ${ }^{9,11}$

ABT-737 (clinical derivative, navitoclax or ABT-263) is a small-molecule inhibitor that binds to the $\mathrm{BH} 3$ domain of

\footnotetext{
${ }^{1}$ Department of Cancer Biology, Lerner Research Institute, Cleveland Clinic, Cleveland, OH 44195, USA; ${ }^{2}$ Department of Pathology, Case Western Reserve University School of Medicine, Cleveland, OH 44106, USA; ${ }^{3}$ Department of Hematology and Oncology, Taussig Cancer Institute, Cleveland Clinic, Cleveland, OH 44195, USA and ${ }^{4}$ Department of Clinical Pathology, Institute of Pathology and Laboratory Medicine, Cleveland Clinic, Cleveland, OH 44195, USA

*Corresponding author: A Almasan, Department of Cancer Biology, Lerner Research Institute, Cleveland Clinic, NB40, 9500 Euclid Avenue, Cleveland, OH 44195, USA; Tel: +1 216444 9970; Fax: +1 216445 6269; E-mail: almasaa@ccf.org

Abbreviations: ABT199-R, ABT-199 resistant; BAX, Bcl-2-associated X protein; BAK, Bcl-2 homologous antagonist/killer; BIM, BCL-2-interacting mediator of cell death; BCL-2, B-cell lymphoma 2; BCL-w, BCL-2-like protein w; Bcl-xL, B-cell lymphoma-extra large; BH3, BCL-2 homology-3; CLL, chronic lymphocytic leukemia; MCL-1, myeloid cell leukemia 1; mTOR, mechanistic target of rapamycin; NOXA, phorbol-12-myristate-13-acetate-induced protein 1; PI, propidium iodide; PI3K, phosphatidylinositol 3-kinase; PUMA, p53 upregulated modulator of apoptosis; qRT-PCR, quantitative real-time polymerase chain reaction; siRNA, small interfering RNA Received 28.7.14; revised 25.10.14; accepted 03.11.14; Edited by M Diederich
} 


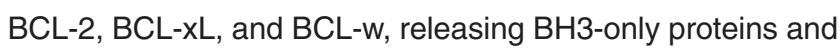
causing mitochondrial outer membrane permeabilization via BAX/BAK activation. ${ }^{12-14}$ Our previous studies with primary CLL samples showed that the inability of ABT-737 to cause cell death in patient-derived samples correlated with high levels of MCL-1 and BFL-1 expression. ${ }^{15}$ Moreover, navitoclax caused on-target toxicity in BCL-xL-dependent platelets, causing thrombocytopenia in CLL patients. ${ }^{16}$ This led to the reengineering of navitoclax into a potent and orally bioavailable BCL-2-specific inhibitor, ABT-199, which demonstrates robust anti-leukemic activity toward BCL-2- but not BCL-xLdependent tumors. ${ }^{17-21}$ Studies with primary patient samples of CLL, acute lymphoblastic leukemia, and mouse xenograft models have shown that prolonged dosing of ABT-199 not only maintains robust antagonism towards BCL-2 but also spares platelets, thus avoiding thrombocytopenia. ${ }^{17,19,22}$

Preliminary data from clinical trials with ABT-199 have shown high response rates in CLL. However, in refractory CLL, initial results of ABT-199 treatment have shown potential for tumor lysis syndrome, requiring slow dose escalation. ${ }^{17,18,23}$ Binding affinity studies with fluorescence polarization assay and TR-FRET showed that ABT-199 has very weak affinity for $B C L-x L$ and $M C L-1$. Correspondingly, cell viability assays with non-Hodgkin lymphoma cell lines have shown that ABT-199 has limited efficacy in BCL-xL- and MCL-1-dependent hematopoietic malignancies. ${ }^{17}$

Acquired and inherent drug resistance is always a potential concern associated with even the most effective chemotherapeutic drugs, impeding their progression in clinical trials for use as single agents. Therefore, here we investigated the mechanisms of ABT-199-resistance in sensitive B-cell lymphoid cell lines after chronic exposure to ABT-199. Our results indicate that acquired ABT-199-R develops because of increased activation of the PI3K/AKT/mTOR signaling pathways and upregulation of $\mathrm{MCL}-1$ and $\mathrm{BCL}-\mathrm{xL}$ that sequester BIM. A combination approach using PI3K inhibitors and ABT-199 sensitized inherent and acquired ABT-199-R cells by targeting critical survival pathways upstream of MCL-1 and $B C L-x L$. Our data reveal novel mechanistic insights into the role of MCL-1 and BCL-xL in ABT-199-resistance and provide rational combination strategies to overcome it in lymphoid malignancies.

\section{Results}

DLBCL cells with low BCL-xL and MCL-1 expression develop resistance to $\mathrm{ABT}-199$ following chronic exposure. ABT-199 has high affinity to bind to BCL-2 at sub-nanomolar concentrations as compared with MCL-1 and $B C L-x L$. Analyzing levels of $B C L-2$ family proteins in representative cell lines from various $B$-cell malignancies showed a high variability in expression, with SU-DHL-6 and OCL-LY-19 cells having low levels of MCL-1 and BCL-XL (Figures $1 \mathrm{a}$ and b). Subsequent cell viability analysis in these two DLBCL cell lines by Annexin V-propidium iodide (PI) assay demonstrated a high sensitivity to ABT-199, with an $\mathrm{IC}_{50}$ of $50 \mathrm{nM}$ for SU-DHL-6 and $70 \mathrm{nM}$ for OCL-LY-19 (Figures 1c and d). To determine whether these sensitive tumor cells would develop ABT-199-resistance in response to chronic ABT-199 exposure, we generated ABT-199-resistant (ABT-199-R) cell lines as described in Materials and Methods. The resistance thus developed was verified by exposing the parental cells, SU-DHL-6, OCL-LY-19, and their resistant derivatives to increasing concentrations of ABT-199 for $48 \mathrm{~h}$ and then analyzing cell viability. Although parental cell lines with low $B C L-x L$ and $M C L-1$ expression were very sensitive to ABT-199, resistant sub-clones were resistant to ABT-199 at concentrations fourfold higher than the $\mathrm{IC}_{50}$ of the parental cells.

ABT-199-R correlates with increase in p-AKT, MCL-1, and BCL-xL levels. To investigate the mechanism of ABT-199resistance, we next examined the expression patterns of BCL-2 family proteins in parental and resistant cells. Immunoblot analyses show that the levels of $\mathrm{MCL}-1$ and BCL-xL were higher in resistant (SU-DHL-6 ABT199-R and OCL-LY-19 ABT199-R) as compared with parental cells (Figure 1e). In contrast to our previous studies of ABT-737resistance in acute lymphoblastic leukemia cell lines, ${ }^{24}$ we found no changes in BCL-2 level between parental and ABT199-R cells. There was also no change in the levels of the BH3-only proteins BIM, NOXA, and PUMA.

We next examined upstream signaling pathways known to control transcription and translation of $\mathrm{MCL}-1$ and $B C L-x L$. Studies on DLBCL have shown that AKT and mTOR, separately or in combination, regulate transcription and/or translation of MCL-1. ${ }^{25-27}$ Exploring AKT activity in our experimental system revealed that $\mathrm{p}-\mathrm{AKT}$ levels were upregulated 1.9-fold in SU-DHL-6 ABT199-R and 1.8-fold in OCLLY-19 ABT199-R cells (Figure 1e). We then tested the effect of acute ABT-199 $(0.75 \mu \mathrm{M})$ treatment on the expression of BCL-2 family members on cell death. Immunoblot analyses indicate that MCL-1 protein level increased from baseline in parental SU-DHL-6, OCL-LY-19, and OCL-LY-19 ABT199-R cells, but not in the SU-DHL-6 ABT199-R derivative cells. In contrast, there were no robust changes in $B C L-x L, B C L-2$, and BIM levels. As expected, caspase-3 was proteolytically cleaved only in parental cells and cleavage occurred as early as $6 \mathrm{~h}$ (Figure 1f, Supplementary Figure S1a), indicating apoptosis activation. NOXA levels increased in SU-DHL-6 only at later time points, with no significant change observed in OCL-LY-19 cells (data not shown). These results indicate that ABT199-R cell express elevated levels of $p-A K T, M C L-1$, and $B C L-x L$ and resist caspase-3 cleavage and thus avoid apoptosis activation. The increase in $\mathrm{MCL}-1$, with no change in $\mathrm{Bcl}-\mathrm{xL}$ levels, in sensitive parental cell lines in response to acute ABT-199 was not sufficient to rescue them from cell death, as indicated by cleaved caspase-3. These findings indicate the importance of $\mathrm{BCL}-\mathrm{xL}$ expression in mediating ABT-199-resistance.

Increased Mcl-1 and Bcl-xL mRNA levels and MCL-1 protein stability contribute to ABT-199-resistance. We next investigated the underlying cause of the increase in MCL-1 and BCL-xL protein levels in ABT199-R cells. qRT-PCR analyses indicated that $M c l-1$ mRNA levels were increased fivefold in SU-DHL-6 ABT199-R and threefold in OCL-LY-19 ABT199-R compared with the respective parental cells. $B c l-x L$ levels were also increased by twofold in 
a
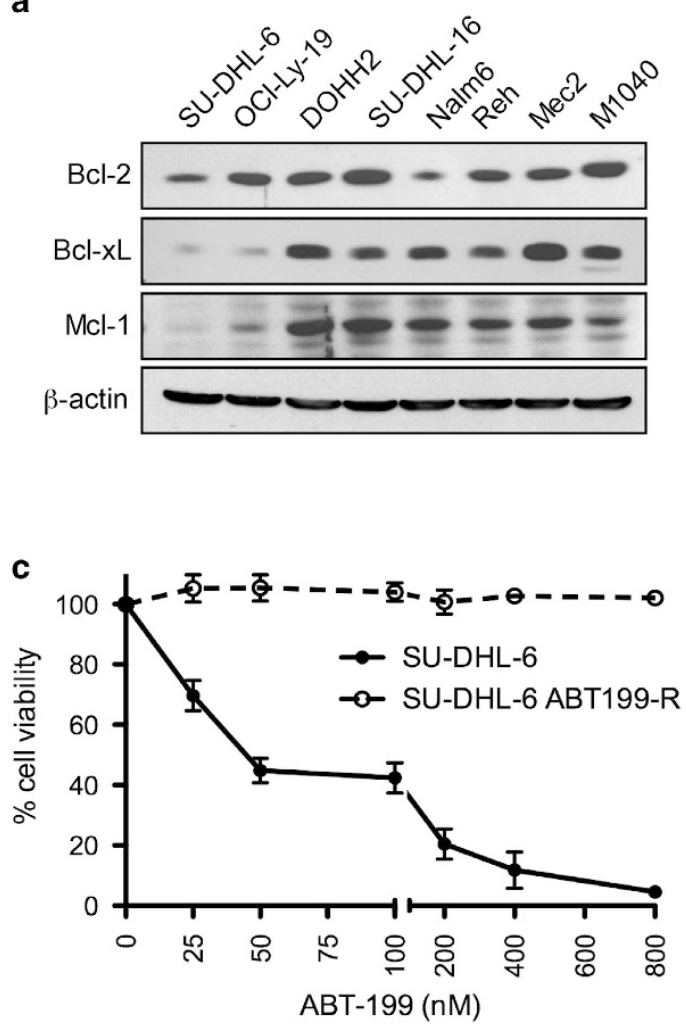

b

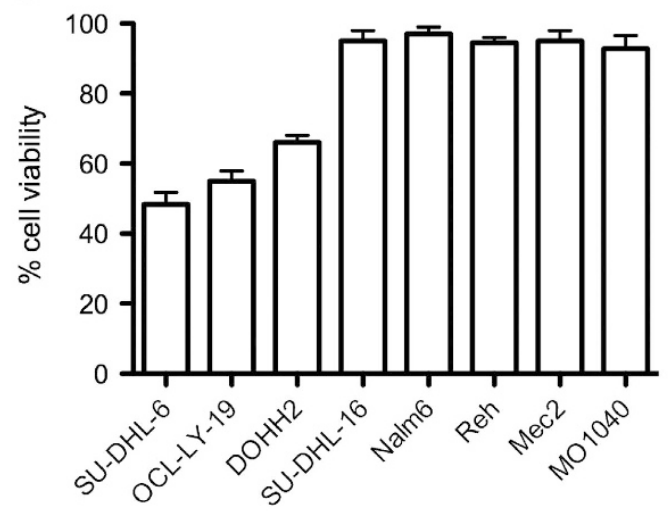

d

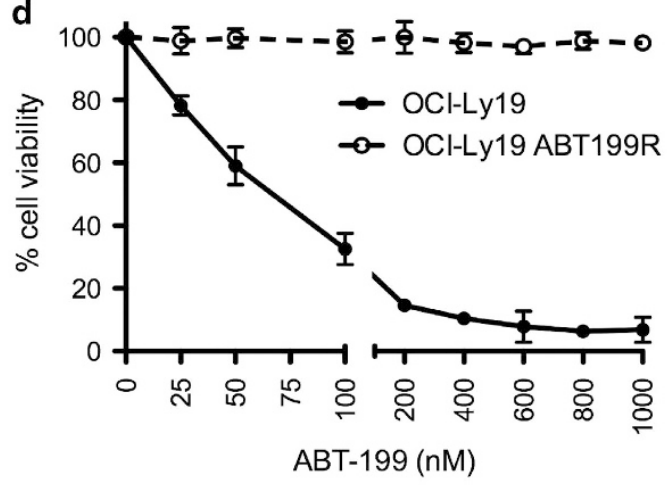

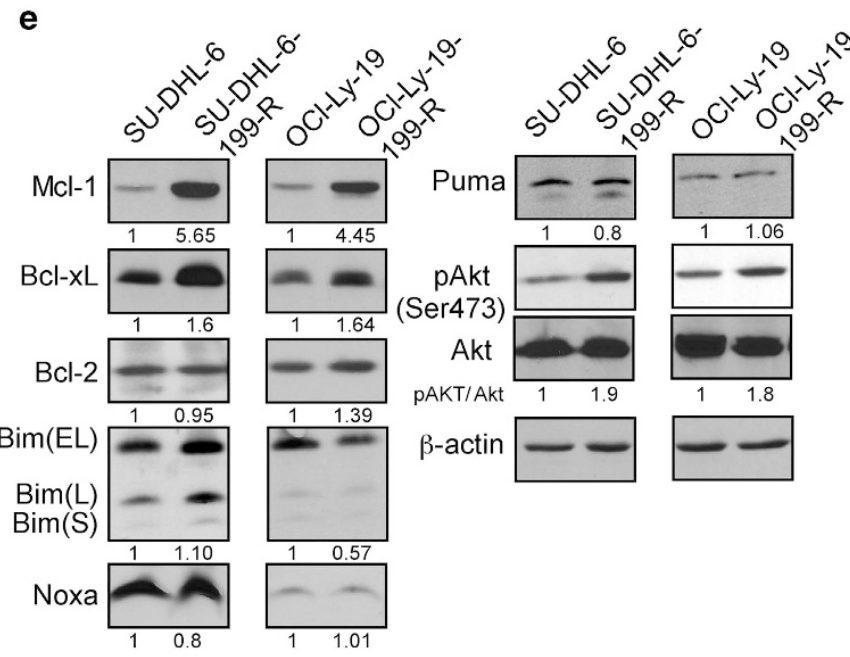

$\mathbf{f}$

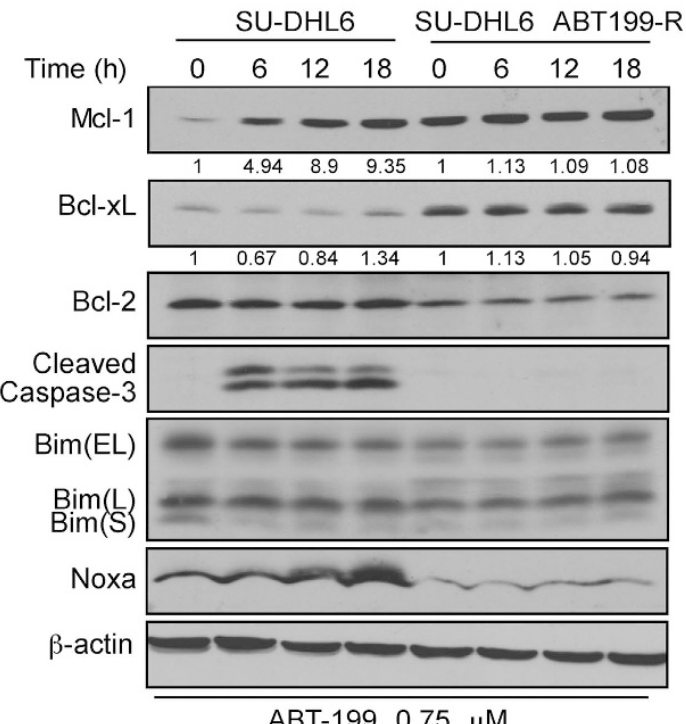

Figure 1 DLBCL cells with low MCL-1 and/or BCL-xL expression are sensitive to ABT-199 and develop resistance after chronic ABT-199 exposure by upregulating MCL-1, BCL-xL, and p-AKT levels. (a) Expression levels of the pro-survival proteins MCL-1, BCL-2, and BCL-xL in DLBCL (SU-DHL-6, OCL-LY-19, SU-DHL-16), follicular lymphoma (DOHH2), acute lymphoblastic leukemia (Nalm6, Reh), and CLL (Mec2, MO1040) cell lines. $\beta$-actin was used as the loading control. (b) The indicated cell lines were treated with $50 \mathrm{nM} \mathrm{ABT-199}$ for $48 \mathrm{~h}$. The viability shown represents the percentage of live cells relative to control cells treated with dimethyl sulfoxide. Parental and ABT-199-R (c) SU-DHL-6 and (d) OCl-Ly-19 cells were treated with the indicated concentrations of ABT-199 for $48 \mathrm{~h}$ and cell viability was determined by Annexin V-PI staining. Control cells were treated with dimethyl sulfoxide. (e) Expression levels of: (i) pro-survival proteins MCL-1, BCL-2, and BCL-xL, (ii) pro-apoptotic proteins BIM, NOXA, and PUMA, and (iii) total AKT and p-AKT (Ser473) in untreated parental and ABT199-R-derivative DLBCL cell lines at the indicated time. SU-DHL-6 parental and resistant cells. Both panels represent one experiment with BCL-2 and $\beta$-actin serving as loading controls. (f) Cells were treated with ABT-199 for the indicated time. MCL-1, BCL-2, BCL-xL, BIM, NOXA, PUMA, and cleaved caspase-3 were determined by immunoblotting. $\beta$-actin was used as a loading control. Standard deviation (S.D.) is indicated in $\mathbf{b}-\mathbf{d}$ as error bars ( $N=3$ ). The experiments in $\mathbf{a}, \mathbf{e}$, and $\mathbf{f}$ are representative of three independent experiments. Numbers below blots indicate increase in protein levels as determined by Image $J$ quantification, which in case of pAKT was normalized to total AKT levels 


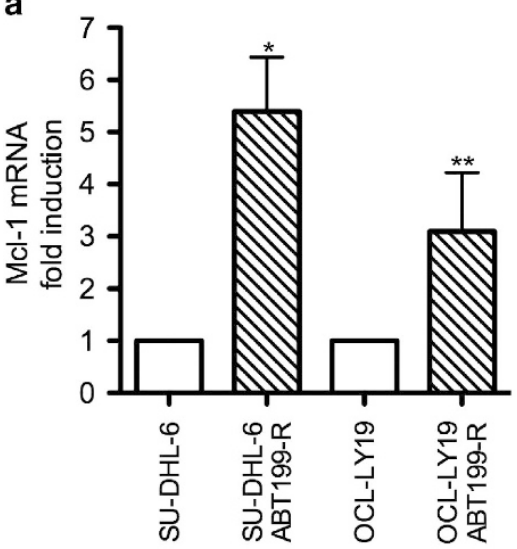

b

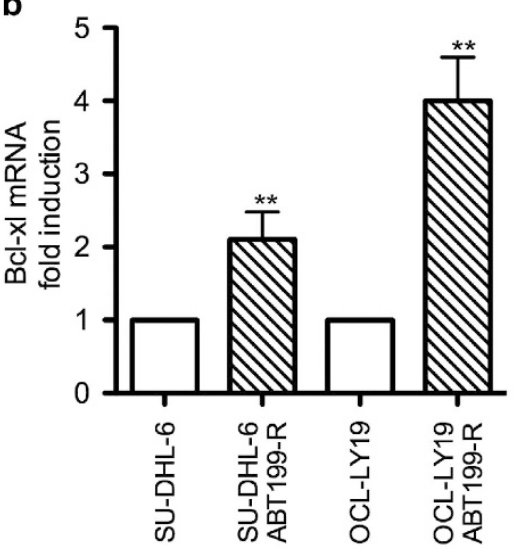

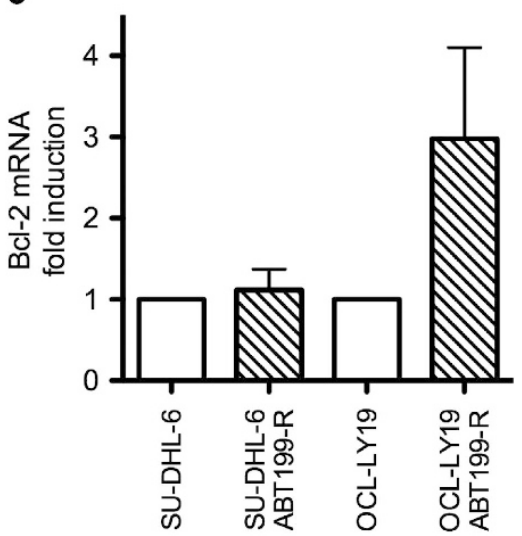

d

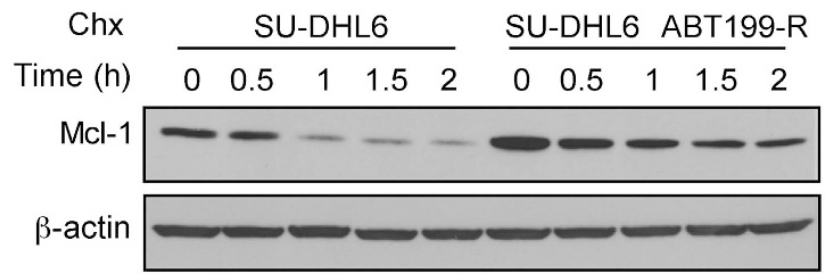

$\mathbf{f}$

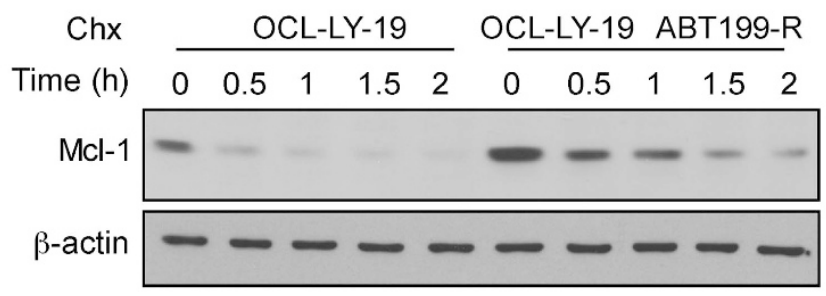

e

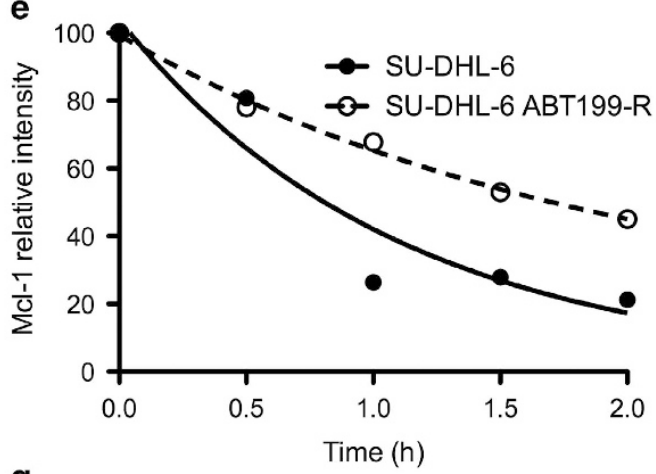

g

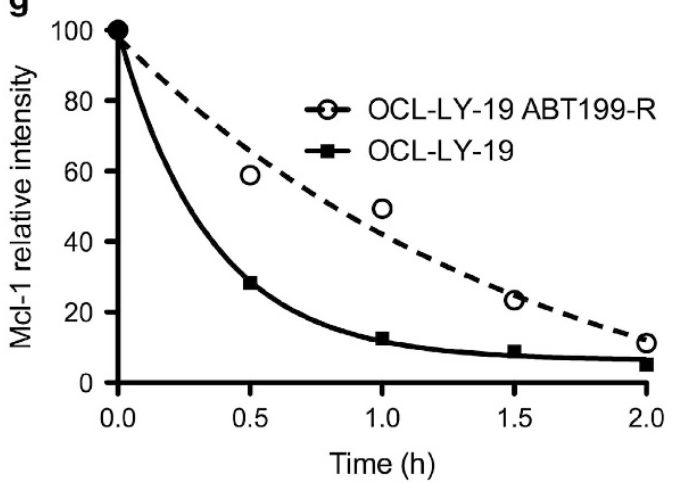

Figure 2 ABT-199-resistance is associated with the upregulation of Mcl-1 and Bcl-xL mRNA and increased MCL-1 protein stability. RNA was extracted from parental and ABT199-R SU-DHL-6 and OCl-LY-19 cells after culture in the absence of ABT-199 for $72 \mathrm{~h}$. (a) Mcl-1, (b) Bcl-xL, and (c) Bcl-2 fold change was analyzed by qRT-PCR. RNA levels of parental cells were set to 1 for analysis $\left({ }^{\star} P<0.04,{ }^{*} P<0.05\right)$. MCL-1 protein half-life was determined by treating parental and ABT199-R (d) SU-DHL-6 and (f) OCI-LY-19 cells with cycloheximide $(10 \mu \mathrm{g} / \mathrm{ml})$ for the indicated time, followed by immunoblotting. $\beta$-actin was used as the loading control. Data in e and $\mathbf{g}$ were quantified by ImageJ. The experiments from a to $\mathbf{g}$ are representative of three independent experiments

SU-DHL-6 ABT199-R and fourfold in OCL-LY-19 ABT199-R cells (Figures $2 \mathrm{a}$ and $\mathrm{b}$ ). Bcl-2 transcript levels were elevated in OCL-LY-19 ABT199-R, but not in SU-DHL-6 ABT199-R cells (Figure 2c). Following a brief incubation with ABT-199 $(0.75 \mu \mathrm{M}), \mathrm{Mcl}-1, \mathrm{Bcl}-x \mathrm{~L}$, and $B c l-2 \mathrm{mRNA}$ levels did not significantly change in parental or resistant SU-DHL-6 and OCL-LY-19 cells (Supplementary Figure S2).

As MCL-1 protein has a short half-life, ${ }^{28}$ we determined its stability by inhibiting its synthesis with cycloheximide, as before. $^{24} \mathrm{MCL}-1$ half-life significantly increased in resistant cells (78 min for SU-DHL-6 ABT199-R and 69 min for OCLLY-19 ABT199-R) as compared with parental cells (42 min for
SU-DHL-6 and 15 min for OCL-LY-19) (Figures 2d and g). Taken together, these results show that elevated MCL-1 and $B C L-x L$ levels in resistant cells could be attributed to increased mRNA levels, as well as MCL-1 protein stability, which developed in the course of acquiring ABT-199-resistance.

Elevated MCL-1 and BCL-xL levels sequester BIM to determine ABT-199-resistance. ABT-199, like its predecessor navitoclax, binds to the $\mathrm{BH} 3$ domain of $\mathrm{BCL}-2$, displacing BIM and causing BAX activation and cell death. ${ }^{17}$ To understand the mechanism of acquired ABT-199-resistance, we next investigated protein-protein interactions between 
pro-survival MCL-1, BCL-xL, and $B C L-2$ proteins and the $\mathrm{BH} 3-$ only protein BIM. MCL-1 was immunoprecipitated from parental and resistant SU-DHL-6 cells lysed with $2 \%$ CHAPS buffer. Immunoblotting analyses show increased BIM binding to MCL-1 in SU-DHL-6 ABT-99-R compared with parental cells (Figure 3a). Similarly, immunoprecipitation of $B C L-x L$ revealed that more BIM was associated with it in ABT199-R cells (Figure $3 b$ ). Reciprocal immunoprecipitation with BIM a

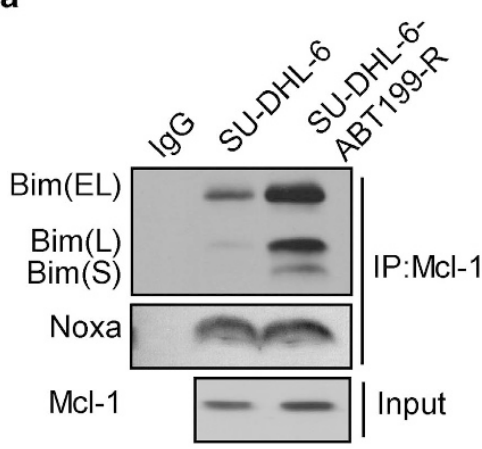

d

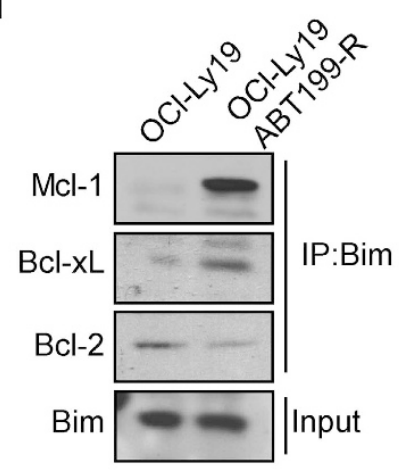

b

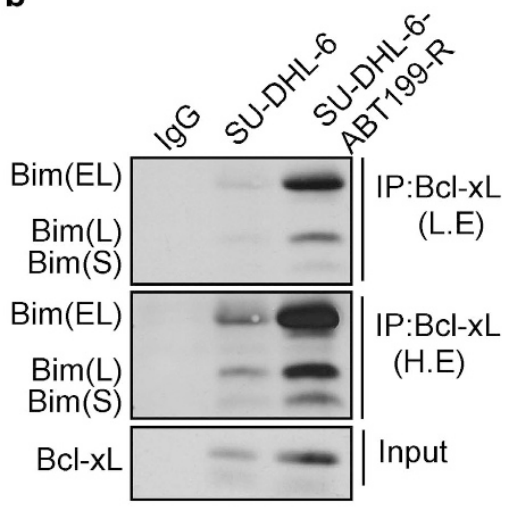

C

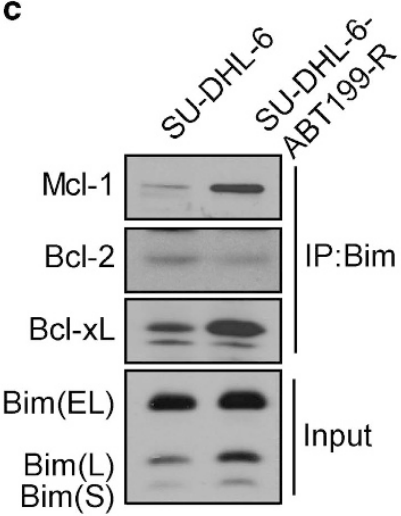

e
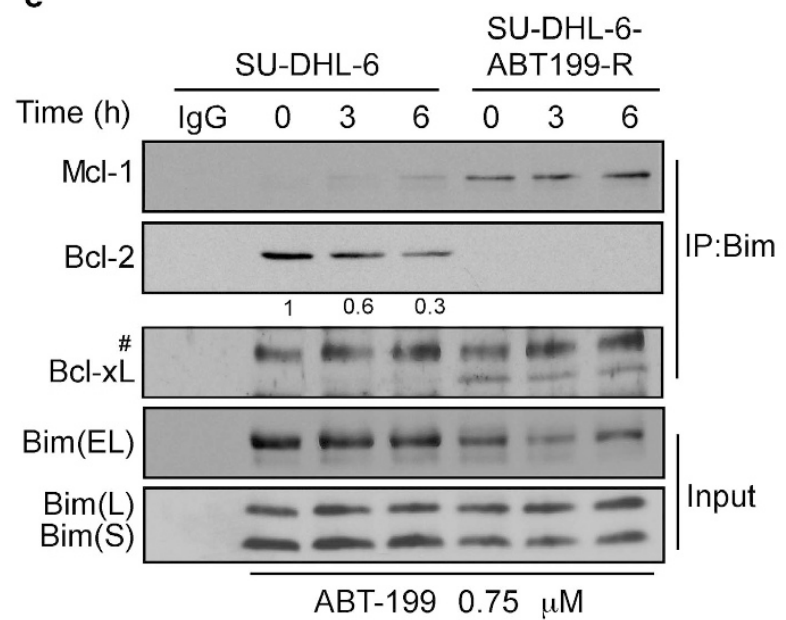

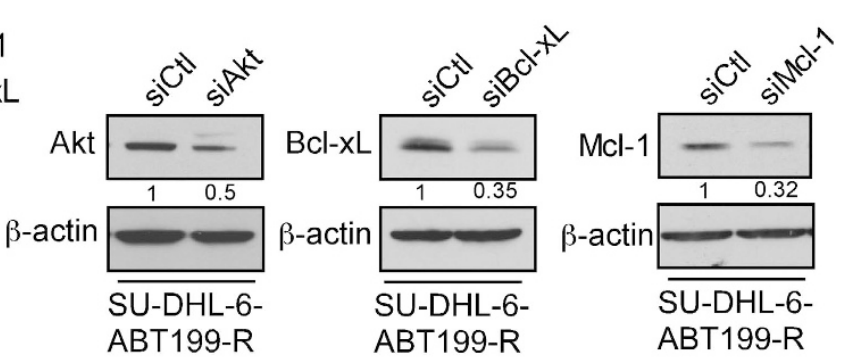

Figure 3 BIM associates with increased MCL-1 and BCL-xL protein in ABT199-R cells. MCL-1 and BCL-XL were immunoprecipitated with (a) MCL-1- and (b) BCL-xL-specific antibodies and their association with BIM and NOXA was probed by immunoblotting with specific antibodies. Reciprocal immunoprecipitation-western blotting for BIM was performed in (c) SU-DHL-6 (d) OCL-LY-19 parental and ABT199-R cell lines and its association with MCL-1, BCL-xL, and BCL-2 was analyzed. (e) Parental and resistant cells were treated with ABT-199 at the indicated time and BIM immunoprecipitates were examined by immunoblotting for association with MCL-1, BCL-xL, and BCL-2 in SU-DHL-6 parental and ABT199-R cells. (f) SU-DHL-6 ABT199-R cells were transfected with siAKT, siMcl-1, siBcl-xL or siControl and treated with the indicated concentration of ABT-199 for $24 \mathrm{~h}$. Cell viability was determined by Annexin V-PI staining. Control cells were treated with dimethyl sulfoxide $\left({ }^{*} P<0.02,{ }^{* \star} P<0.001\right)(N=2)$. $\beta$-actin was used as loading control. The experiments from a to e are representative of three independent experiments. L.E and H.E: low and high exposure, respectively. \#, non-specific band 
and immunoblotting for BCL-2, MCL-1, and BCL-xL confirmed these observations. As expected, more MCL-1 and $\mathrm{BCL}-\mathrm{xL}$ was associated with $\mathrm{BIM}$ in the resistant compared with parental cells (Figure $3 \mathrm{c}$ ). Strikingly, less BCL-2 was bound to BIM in the resistant cells. Similar results were observed in immunoprecipitation studies performed in OCLLY-19 parental and resistant cells (Figure 3d). Moreover, BIM immunoprecipitation from lysates of SU-DHL-6 parental and resistant cells that had undergone acute ABT-199 exposure revealed that BIM was displaced from BCL-2 by ABT-199 only in parental, but not in ABT199-R cells (Figure $3 e$ and Supplementary Figure S1b). Taken together, these results show that elevated MCL-1 and BCL-xL levels sequester BIM that was displaced from BCL-2 during the course of acquired ABT-199-resistance. This property allowed the resistant cells to avoid the dependency on BCL-2 that is exploited by ABT-199 in parental cells. To directly demonstrate a role for MCL-1, BCL-xL, and AKT, we reduced their levels by the respective siRNAs in SU-DHL-6 ABT199-R cells (Figure 3f). Annexin V-PI analysis following ABT-199 treatment showed that two $(A K T)$ to threefold (MCL-1 and $B C L-x L)$ reduction in protein levels increased the sensitivity of ABT-199R cells significantly. These findings indicate that: (i) activation of the AKT pathway, and (ii) upregulation of $M C L-1$ and $B C L-x L$ are key facets in mediating ABT-199-resistance.

NVP-BEZ235, in combination with ABT-199, downregulates MCL-1 levels and sensitizes ABT199-R cells. The above data establish the important role of $\mathrm{MCL}-1$ and $\mathrm{BCL}-\mathrm{xL}$ in mediating ABT-199-resistance. In order to overcome acquired as well as inherent ABT199 resistance, we next selected clinically relevant pharmacological inhibitors that could target signaling pathways responsible for maintaining high MCL-1 levels. Because both p-AKT and mTOR have been reported to affect $\mathrm{MCL}-1$ levels, ${ }^{27,29,30}$ we targeted these pathways with a dual $\mathrm{PI} 3 \mathrm{~K} / \mathrm{mTOR}$ inhibitor, NVPBEZ235, in combination with ABT-199.

Cell viability analysis by Annexin V-PI showed high sensitivity to a $24 \mathrm{~h}$ incubation with the ABT199-NVP+ BEZ235 combination, whether ABT-199-R was acquired (SU-DHL-6 ABT199-R and OCL-LY-19 ABT199-R) or inherent $(\mathrm{DOHH} 2, \mathrm{SU}-\mathrm{DHL}-16)$. In contrast, treatment with either agent alone was not effective (Figures $4 \mathrm{~b}$ and e). Moreover, no additional cell death occurred with this combination treatment in parental cell lines when compared with ABT-199 treatment alone (Figure $4 \mathrm{a}$ ). As this combination induced maximum cell death at $24 \mathrm{~h}$, an earlier $6 \mathrm{~h}$ time point was further chosen to study the mechanism of action of this combination treatment. ABT199-R cells were treated with ABT-199 (400 nM) and NVP-BEZ235 (40 nM), alone or in combination. Interestingly, in ABT199-R cells, MCL-1 expression, which had declined after NVP-BEZ235 treatment alone, further decreased with the combination treatment, despite the fact that ABT-199 alone increased MCL-1 levels (Figures 1e, f, and 5a). In contrast, $B C L-2$ and $B C L-x L$ levels did not change. In accordance with the cell viability data, caspase-3 was activated only following the combination treatment, suggesting that resistant cells depended on MCL-1 for their survival.

Evaluation of signaling proteins in the PI3K/mTOR pathway showed a decrease in active AKT that was more pronounced following the ABT-199+NVP-BEZ235 combination. Because NVP-BEZ235 also targets mTOR, we next examined the activity of the downstream targets of mTORC1. Phosphorylation of p-70S6 kinase and 4-EBP1 was inhibited not only after treatment with NVP-BEZ235 alone but also when it was used in combination with ABT-199. Total AKT and p-70S6 kinase levels did not significantly change (Figure 5a). These findings indicate that a decrease in $\mathrm{MCL}-1$ protein expression in response to the ABT-199+NVP-BEZ235 combination was associated with decreased activity of AKT and mTOR. Next, we investigated the mechanism of sensitization by this combination by examining the association between MCL-1 or BCL-xL and BIM. MCL-1 immunoprecipitation revealed that following the combination treatment, it was no longer associated with BIM (Figure 5b), and BIM and NOXA expression was not changed. The reciprocal immunoprecipitation with BIM indicated a decreased association of BIM with MCL-1 (Figure 5c). The BIM association with BCL-xL was decreased after NVP-BEZ235-only treatment that was further reduced with the ABT-199 and NVP-BEZ235 combination.

It has been reported in $\mathrm{Bax}^{-1-}, \mathrm{Bak}^{-1-}$ mouse embryonic fibroblasts $^{17}$ that ABT-199 is ineffective, because Bax and Bak are essential for activating apoptosis. ${ }^{31}$ Acute ABT-199 treatment led to BAX activation in parental SU-DHL- 6 but not in ABT199-R-derivative cells. These results indicate that $B A X$ activation in response to ABT-199 is impaired in ABT-199-R cells (Figure $5 d$ ). Interestingly, BAX was activated with the ABT-199+NVP-BEZ235 combination in ABT199-R cells, but not with the individual treatments, corresponding to a decrease in MCL-1 levels (Figures $5 \mathrm{a}$ and $\mathrm{d}$ ). These findings further highlight the resistant cell's dependency on MCL- 1 to avoid BAX activation. Because NOXA level did not increase with the combination treatment in ABT199-R cells, we can conclude that disruption of the BIM/MCL-1 association was not due to an increase in NOXA/MCL-1 association, but was rather a result of a decrease in PI3K/mTOR activity and downregulation of MCL-1. The inhibition of the PI3K/ mTOR pathway by NVP-BEZ235 alone was not sufficient to cause cell death; however, it potentiated the effect of ABT-199.

ABT-199 in combination with GS-1101 sensitizes ABT-199-R cells by targeting the PI3K pathway. Given that the dependency of ABT-199-R cells on MCL-1 is regulated by the $\mathrm{PI} 3 \mathrm{~K} / \mathrm{mTOR}$ pathway, we next asked whether these cells could be sensitized to ABT-199 by targeting the PI3K or mTOR pathways. We used the PI3K $\delta$ and mTORC1 inhibitors, GS-1101 and RAD001, respectively, as before, ${ }^{32,33}$ in combination with ABT-199. Cell viability analyses with Annexin V-PI indicated that GS-1101 in combination with ABT-199 sensitized SU-DHL-6 ABT199$R$ cells more than the RAD001+ABT-199 combination (Figures $6 a$ and $d$ ). The latter combination was effective only at a higher concentration of RAD001 that may not be achieved in clinical settings. Similar results were found in OCL-LY-19 ABT199-R and inherently resistant DOHH2 cells (Figures $6 e$ and $f$ and data not shown). Individual treatment with RAD001 and GS-1101 had no effect on parental 
a

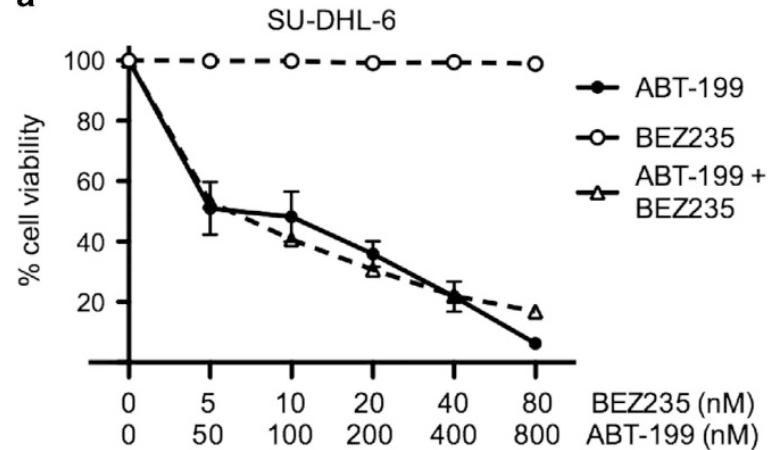

b

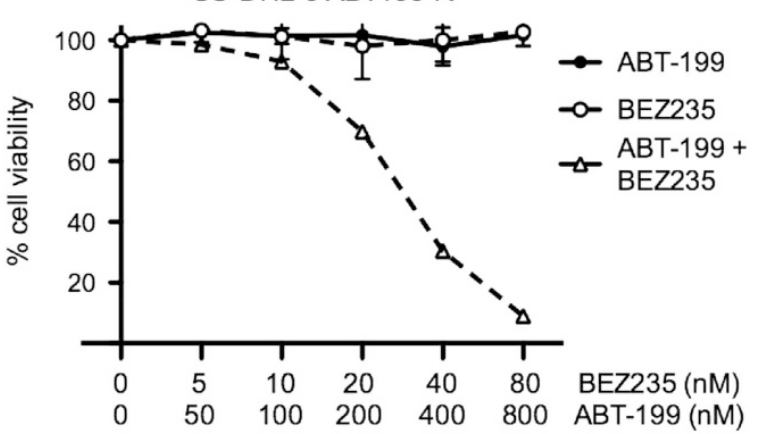

C

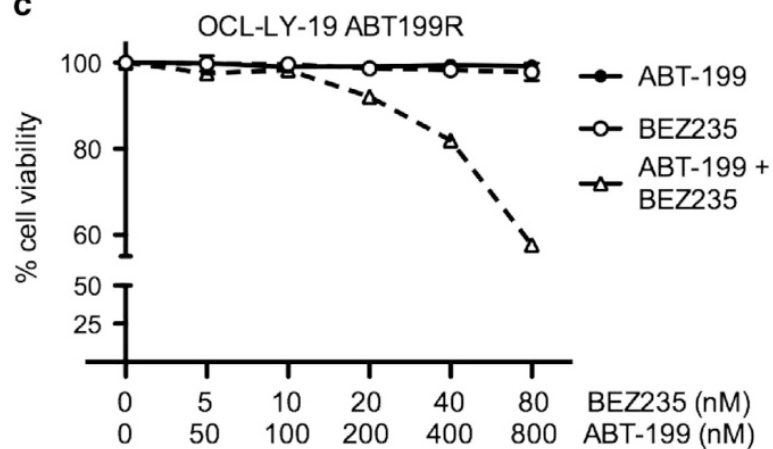

d

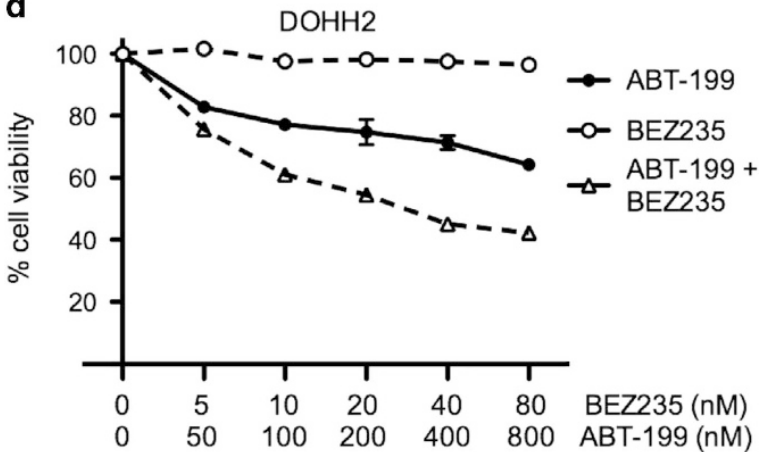

e

SU-DHL-16

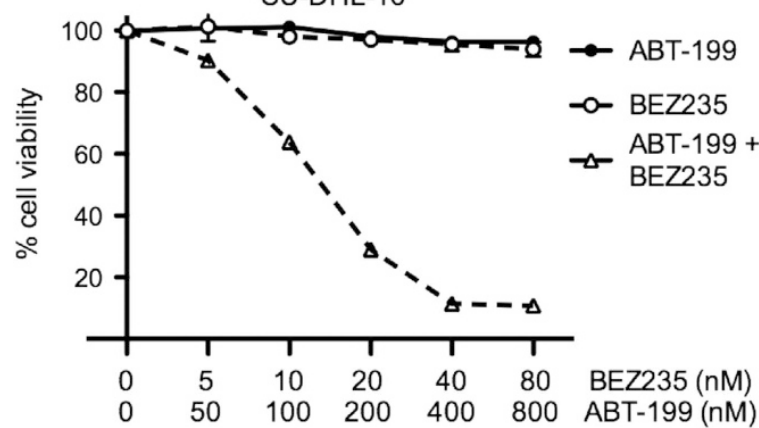

Figure 4 ABT-199 in combination with NVP-BEZ235 sensitizes ABT199-R cells. Parental and resistant derivative DLBCL cell lines (a) SU-DHL-6 (b) SU-DHL-6 ABT199-R (c) OCI-Ly-19 ABT-199R (e) SU-DHL-16, and (d) FL cell line DOHH2 were treated with the indicated concentration of ABT-199 and NVP-BEZ235, alone and in combination for $24 \mathrm{~h}$. Cell viability was determined by Annexin V-PI staining represented as percentage relative to control cells treated with dimethyl sulfoxide. Standard deviation (S.D.) is indicated by the error bars $(N=3)$

and a moderate effect on resistant cells at the higher concentrations.

To gain a better understanding of the signaling proteins that are responsible for the GS-1101+ABT-199 sensitization, we treated SU-DHL-6 and OCL-LY-19 ABT199-R cells with ABT-199+GS-1101 for $24 \mathrm{~h}$. Immunoblotting analysis show decreased $\mathrm{MCL}-1$ expression with this combination, with no changes observed in $\mathrm{BCL}-2$ and $\mathrm{BCL}-\mathrm{xL}$ expression levels. The decrease in $\mathrm{MCL}-1$ corresponded to an increase in cleaved caspase-3 (Figure 6g). AKT activity decreased following GS-1101 addition alone, which was more pronounced with the ABT-199 combination in resistant cells. BAX was activated in ABT199-R cells only with the combination treatment (Figure 6f). Taken together, these findings indicate that the GS-1101+ABT-199 combination exploited the resistant cell's dependency on the $\mathrm{PI} 3 \mathrm{~K} / \mathrm{mTOR}$ pathway by decreasing MCL-1 expression through regulating AKT activity to cause apoptosis (Figure 7).

\section{Discussion}

Rational drug development must anticipate potential mechanisms of resistance, both intrinsic and acquired, in order to develop biomarkers to predict patient response, stratify patients, and to design combination regimens with increased efficacy and applicability. Here, we have addressed the emerging concern that resistance develops to novel $\mathrm{BH} 3$ mimetics in lymphoid malignancies and have demonstrated rational combination approaches to overcome it based on the resistant determinants developed. Navitoclax, a small molecule $\mathrm{BH} 3$ mimetic, was designed to function as a dual inhibitor against BCL-2 and BCL-xL in refractory CLL. ${ }^{11,16,24} B C L-x L$ 


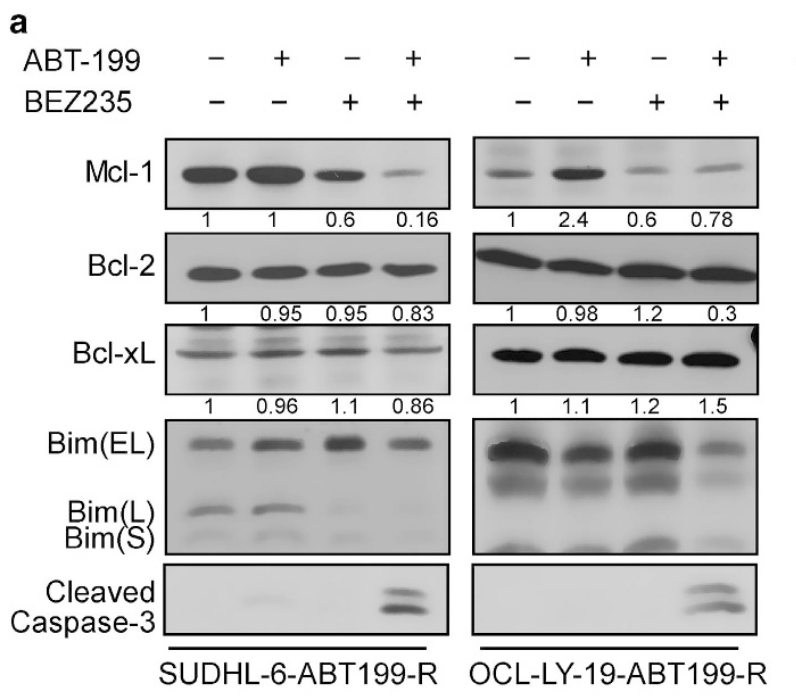

b

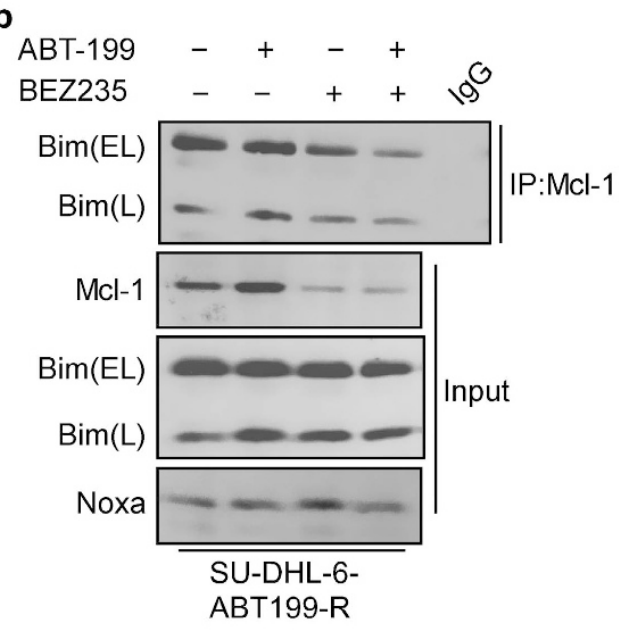

C

d
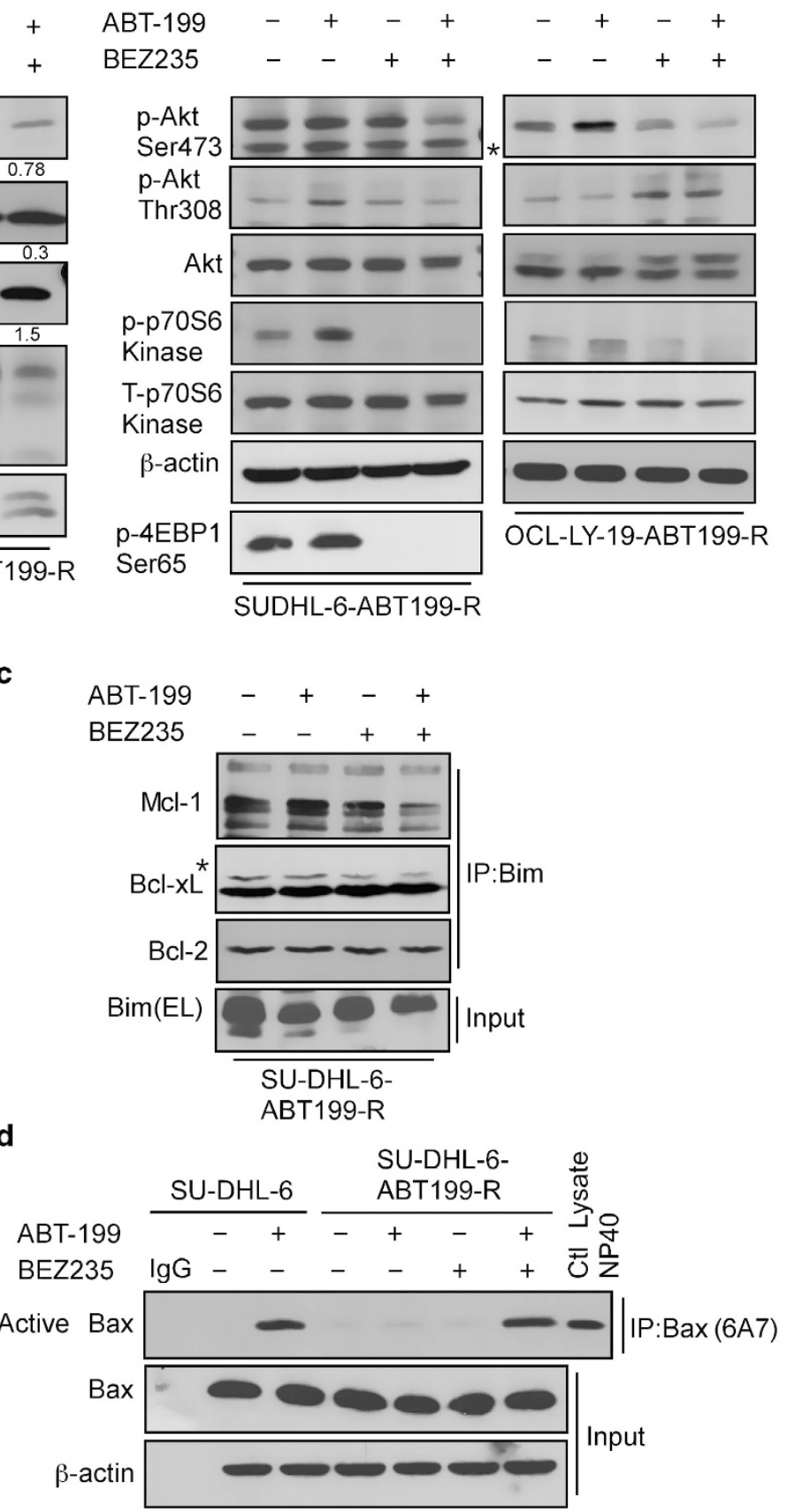

Figure 5 NVP-BEZ235 downregulates MCL-1 through p-AKT and mTOR inhibition and causes BAX activation following BIM release from MCL-1. (a) Expression of MCL-1, BCL-XL, BCL-2, BIM, p-AKT (Ser473), AKT, p-p70S6 kinase (Thr389), p-70S6 kinase, p-4EBP1 (Ser65), and cleaved caspase-3 in SU-DHL-6 ABT199-R and OCL-LY-19 ABT199-R cells treated with ABT-199 (400 nM), NVP-BEZ235 (40 nM) or in combination for $6 \mathrm{~h}$. Both panels represent one experiment with BCL-2 and $\beta$-actin serving as loading controls. * denotes a non-specific band. SU-DHL-6 ABT199-R cells were treated with ABT-199R or NVP-BEZ235, alone or in combination, in concentrations as in a for $6 \mathrm{~h}$ and cells were lysed with $2 \%$ CHAPS buffer. (b) MCL-1 and (c) BIM were immunoprecipiated and their corresponding binding partners MCL-1, BIM, NOXA, BCL-2, or BCL-XL were analyzed by western blotting with specific antibodies. (d) Parental and resistant SU-DHL-6 cells treated with ABT-199R, NVP-BEZ235, or their combination (concentration as in a) were lysed with $1 \%$ CHAPS buffer and active BAX was immunoprecipated with BAX $6 A 7$ and probed by BAX N20 antibodies by immunoblotting. The experiments in a to $\mathrm{d}$ are representative of three independent experiments

inhibition in clinical settings led to dose-dependent thrombocytopenia that prompted redesigning of navitoclax into a BCL-2-specific inhibitor, ABT-199. This second-generation inhibitor has a strong affinity for BCL-2, with no effect on human platelets. ${ }^{17}$ Our results show that as a single agent,
ABT-199 effectively induced apoptosis in DLBCL cell lines in the nanomolar concentration range as early as $4 \mathrm{~h}$ after treatment. Similar results were reported for AML primary cells and mouse models. ${ }^{19}$ However, ABT-199 was less effective against cell lines that had high intrinsic MCL-1 and $B C L-x L$ 

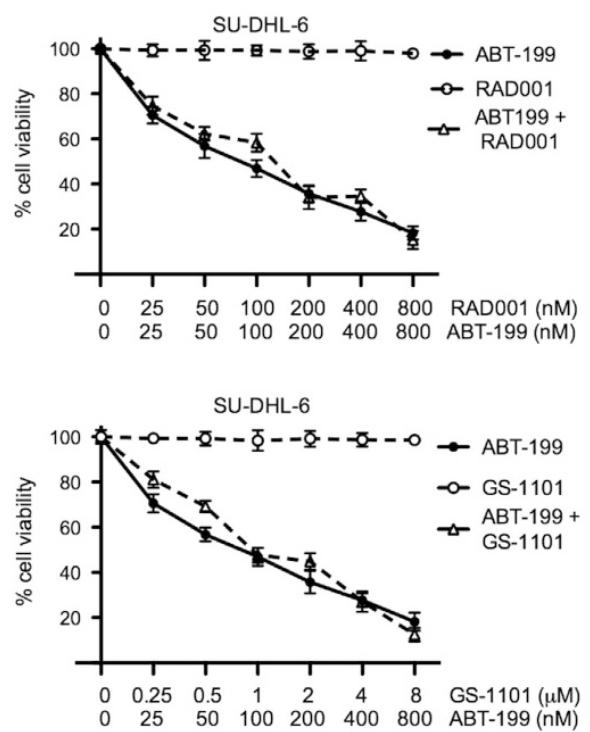

e

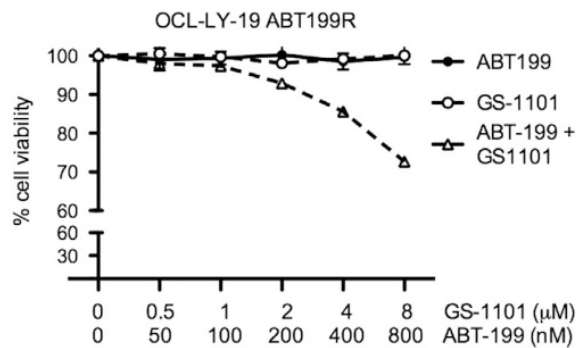

g

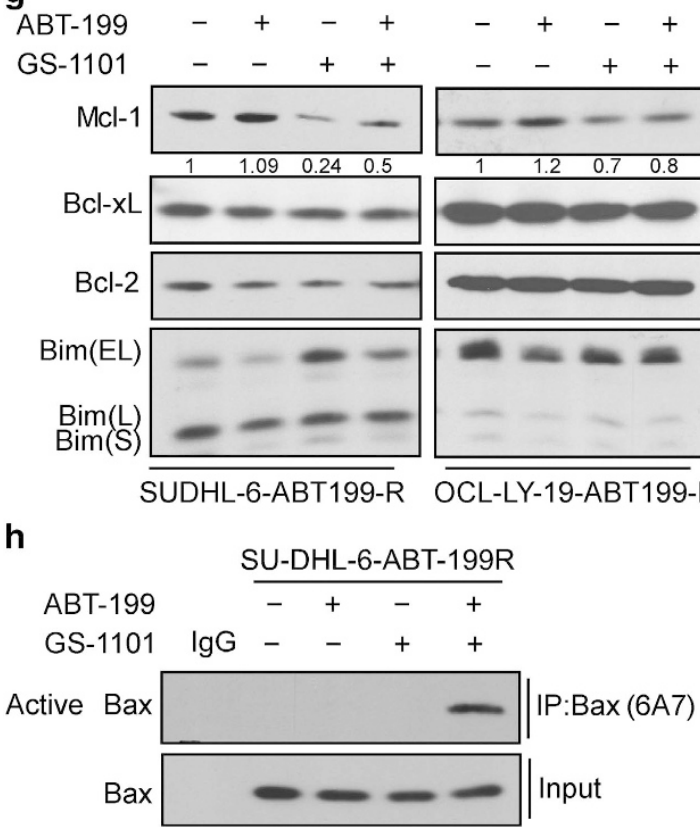

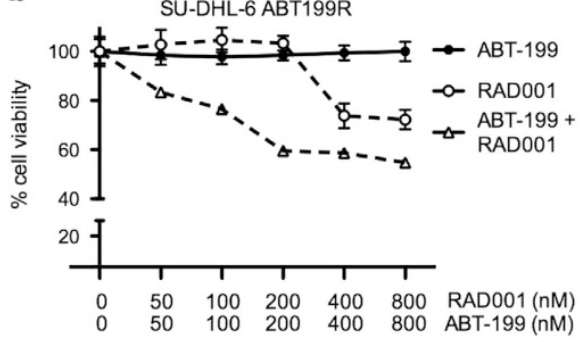

d

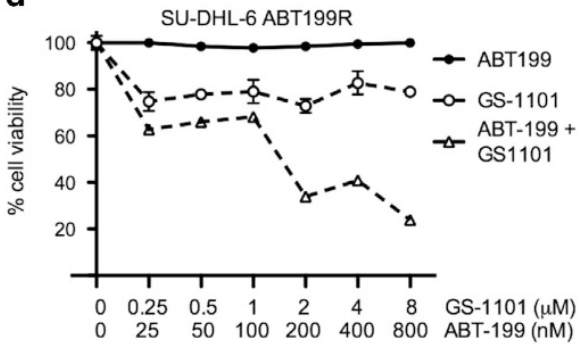

f
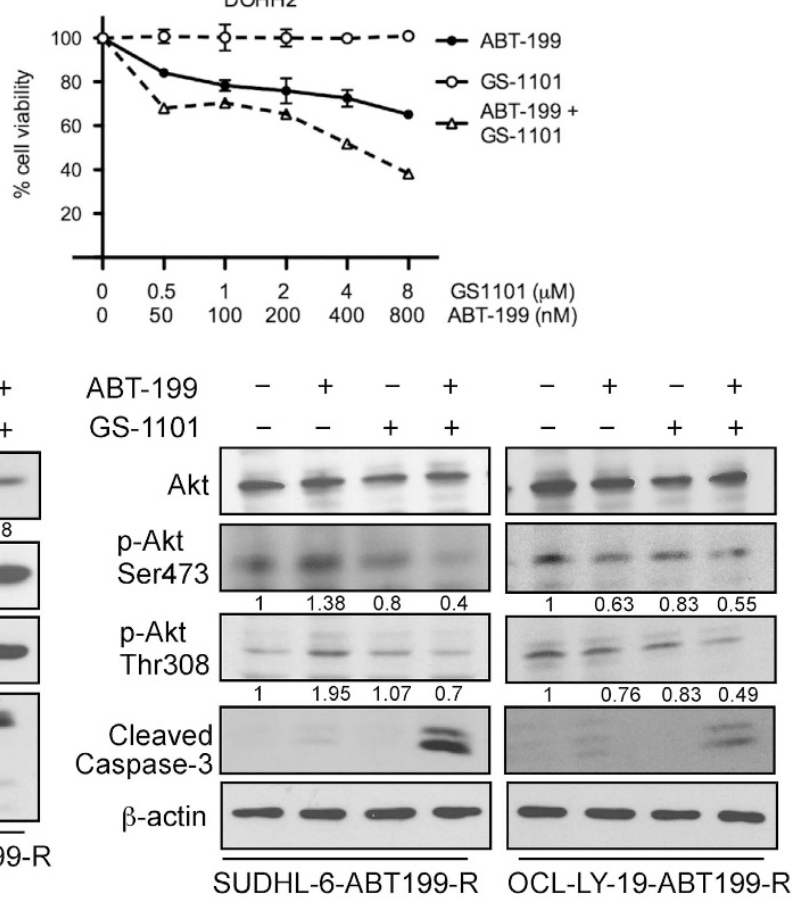

Figure 6 GS-1101 in combination with ABT-199 targets the PI3K pathway, sensitizing ABT-199-R cells by MCL-1 downregulation and BAX activation. Parental and ABT-199R-derivative DLBCL cell lines SU-DHL-6 (a-d), OCL-LY-19 ABT199-R (e) and the FL cell line DOHH2 (f) were treated for $24 \mathrm{~h}$ with the indicated concentration of ABT-199, RAD001, and GS-1101, alone or in combination. Cell viability was determined by staining with Annexin V-PI, and represented as the percentage relative to control cells treated with dimethyl sulfoxide. Standard deviation (S.D.) is indicated in a-e by error bars $(N=3)$. (g) Expression levels of MCL-1, BCL-xL, BCL-2, BIM, p-AKT (Ser473), p-AKT (Thr308), AKT, and cleaved caspase-3 in SU-DHL-6 ABT199-R cells treated with ABT-199 (400 nM) and GS-1101 $(4 \mu \mathrm{M})$ alone or in combination, and OCL-LY-19 ABT199-R treated with ABT-199 (400 nM) and GS-1101 (8 $\mu$ M), alone or in combination, for $24 \mathrm{~h}$. $\beta$-actin was used as the loading control. (h) ABT-199-R SU-DHL-6 cells treated with ABT-199R $(400 \mathrm{nM})$, GS-1101 $(4 \mu \mathrm{M})$, alone or in combination, were lysed with 1\% CHAPS buffer, and active BAX was immunoprecipated with BAX 6A7 and detected using BAX N20 antibodies following immunoblotting. The experiments in $\mathbf{g}$ and $\mathbf{h}$ are representative of three independent experiments 
a

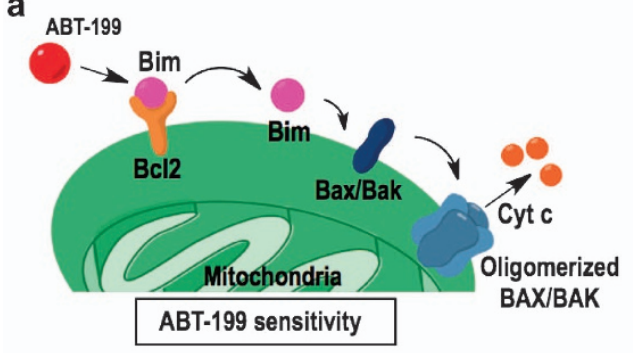

b

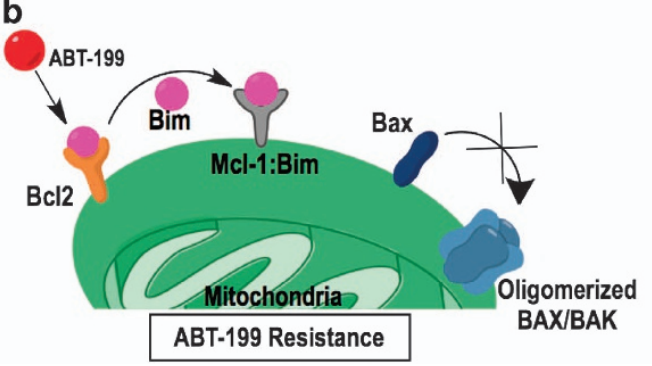

C

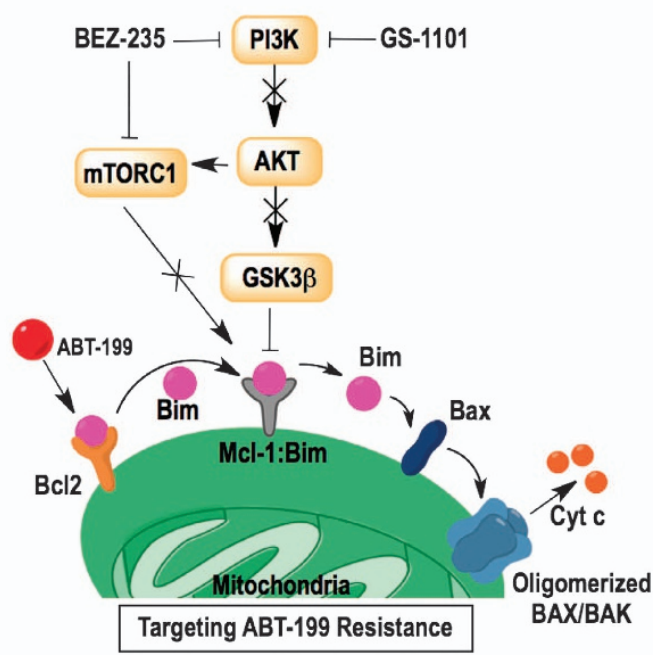

Figure 7 Model for ABT-199-resistance and PI3K-mTOR-mediated targeting of ABT-199-R cells. (a) ABT-199 targets BCL-2 in sensitive cells and displaces BIM to cause apoptosis through BAX activation. (b) ABT-199 does not target MCL-1 and BCL-xL, which confers resistance by sequestering BIM displaced from BCL-2. (c) NVP-BEZ235 inhibits the PI3K and mTOR pathways, which interfere with MCL-1 stability, thereby freeing BIM, which then activates BAX, leading to release of cytochrome $c$ to cause cell death

expression. In our efforts to understand the mechanism of ABT-199-resistance, we developed ABT-199-R variants from ABT-199-sensitive DLBCL cell lines. On comparing expression of BCL-2 family members between ABT199-R and parental cells, we found that the constitutive expression of MCL-1 and BCL-xL was a key feature in maintaining ABT-199resistance.

MCL-1, an essential cellular protein, ${ }^{34}$ has a short half-life that distinguishes it from BCL-2 and BCL-xL. The half-life of MCL-1 depends on the phosphorylation of residues on its PEST domain that determines its enhanced ability to sequester BIM and also regulates its proteasomal-mediated degradation. ${ }^{28}$ In addition to increased steady state $\mathrm{Mcl}-1$ and $B c l-x L$ mRNA levels, MCL-1 protein was also more stable in ABT199-R cells. BIM was predominantly associated with BCL-2 in parental cells, which made them sensitive to ABT-199. In contrast, the high MCL-1 and BCL-xL expression in ABT199-R cells sequestered BIM and made them resistant to chronic ABT-199 exposure (Figure 7). A recent study has reported the presence of mutations in BCL-2 and $B A X$ in $A B T-199-R$ mouse and human cell lines, respectively, that conferred ABT-199-resistance. ${ }^{35}$ Although we did not explore the mutational status in our resistance model, the fact that BIM was associated with MCL-1 and $B C L-x L$, but not BCL-2, indicates that MCL-1 and BCL-xL play a major role in $A B T-199$ resistance. Moreover, BIM binding to $\mathrm{MCL}-1$ and $\mathrm{BCL}-\mathrm{xL}$ in ABT199-R cells did not change either in the presence or absence of $A B T-199$, indicating that $A B T-199$-resistance was irreversible. It should be noted that with acute ABT-199 treatment $M C L-1$, but not $B C L-x L$, level was increased in parental cells. However, this transient increase in MCL-1 level could not capture and sequester BIM that ABT-199 displaced from BCL-2, nor could it prevent cell death by BAX activation and cleavage of caspase-3. These findings indicate the importance of $\mathrm{BCL}-\mathrm{xL}$ along with $\mathrm{MCL}-1$ in mediating ABT-199-resistance.

B-cells, circulating and those residing in the lymph node niche, receive critical survival, adhesion, and proliferative signals from B-cell receptor signaling through the PI3K/AKT/ mTOR pathway. These signals are specifically mediated by the delta isoform (PI3K $\delta$ ) of PI3K that is mainly expressed on $\mathrm{B}$ cells. $^{8,26,36,37}$ The PI3K/AKT/mTOR pathways regulate MCL-1 by 4 EBP $1 / m$ TORC1-dependent translation, AKT/GSK3- $\beta$ dependent ubiquitination followed by proteasomal-mediated degradation, and AKT/STAT3- or AKT/CREB-mediated transcription. ${ }^{9,25,38-40}$ Hence, we examined whether the apoptotic potential of ABT-199 would be enhanced by combining it with PI3K/AKT/mTOR inhibitors, particularly in cells with elevated MCL-1 and $B C L-x L$ levels. We recently reported that malignant $B$ cells resistant to fludarabine have increased mTOR activity that can be effectively targeted to overcome their resistance. ${ }^{33}$ AKT activation was, indeed, elevated in ABT199-R cells, thus making them more sensitive to simultaneous inhibition of the PI3K/AKT pathway (by the $\mathrm{PI3K} / \mathrm{AKT} / \mathrm{mTOR}$ inhibitor NVP-BEZ235 or the PI3K $\delta$ inhibitor GS-1101) and BCL-2 (by ABT-199). Similar results were obtained with a combination approach in cells that were inherently resistant to ABT-199 owing to their high MCL-1 and $B C L-x L$ protein expression.

Mechanistically, NVP-BEZ235+ABT199 and GS-1101+ ABT199 treatments could downregulate MCL-1, releasing BIM sequestered by MCL-1 and leading to BAX activation (Figure 7). This approach provides a more clinically amenable and effective alternative to using pan-BCL-2 family inhibitors, such as obatoclax or gossypol, ${ }^{24,41}$ or specific inhibitors on MCL-1, such as maritoclax ${ }^{42}$ or UMI-77. ${ }^{43}$ In vivo experiments with GS-1101 have shown that it can block the B-cell receptor signaling pathway by inhibiting PI3K and preventing activation of its downstream targets AKT and ERK. ${ }^{36,44}$ In addition to the 
potential intracellular benefits of this combination, clinically, the GS-1101+ABT-199 combination could have another benefit. In the absence of critical signals via the B-cell receptor, CLL cells mobilize into the blood stream, where they may be more 'primed for death'36 and hence would be sensitive to ABT-199. A similar effect can be expected for NVPBEZ235, which inhibits both PI3K and mTOR (mTORC1 and mTORC2) pathways. In fact, this dual inhibition also targets feedback signaling that regulates AKT activation. Indeed, cell death in response to NVP-BEZ235+ABT199 was more robust with inhibition of AKT and mTORC1 (as seen by evaluating its downstream targets $\mathrm{p}-4 \mathrm{EBP} 1$ and $\mathrm{p}-\mathrm{p} 70 \mathrm{~S} 6$ kinase) and corresponding downregulation of $\mathrm{MCL}-1$. Interestingly, the combination treatment did not lower BCL-xL expression in ABT199-R cells, which would be beneficial in the clinic, as it would probably not lead to thrombocytopenia in patients as reported with navitoclax. ${ }^{16}$ It should be noted that although both $B C L-x L$ and $M C L-1$ were required for developing acquired ABT-199-R, targeting only one sensitized resistant cells to ABT-199. Suppression of MCL-1 expression with PI3K/ AKT/mTOR inhibitors was not, however, sufficient to tip the balance toward cell death unless ABT-199 was added.

In summary, combining PI3K/AKT/mTOR inhibitors with ABT-199 disturbed the critical equilibrium between antiapoptotic and BH3-only proteins, lowering the threshold needed to activate apoptosis. Our findings provide new insights into the molecular mechanisms of ABT-199resistance and indicate the potential for combining ABT-199 with PI3K/AKT/mTORC inhibitors to lower ABT-199 dose and enhance its applicability in solid tumors as well.

\section{Materials and Methods}

Cell lines and reagents. Human leukemic cell lines SU-DHL-6, OCL-LY-19, SU-DHL-16 (DLBCL), and DOHH2 (FL) were obtained from Dr. Eric Hsi and Dr. Neetu Gupta (Cleveland Clinic), Nalm6, Reh (acute lymphoblastic leukemia) were purchased from the American Type Culture Collection (Manassas, VA, USA), Mec-2 from Dr. Y. Saunthararajah (Cleveland Clinic) and M01040 (both CLL) from Dr. Riccardo Dalla-Favera (Columbia University) and used as before. ${ }^{32,33,45}$ All cell lines were cultured in RPMI-1640 medium supplemented with 10\% FBS (Atlanta Biologicals, Lawrenceville, GA, USA), and antibiotic-antimycotic (Gibco, Life Technologies, Gaithersburg, MD, USA). ABT199-R cells were cultured with $5 \%$ FBS. Cell lines were routinely screened for Mycoplasma, variations in growth rates, changes in morphological characteristics, and their response to stress with Annexin V FITC-PI staining; their passage number did not exceed 20. ABT-737 and ABT-199 were obtained from AbbVie (Chicago, IL, USA); NVP-BEZ235, RAD001, and GS-1101 from Selleck Chemicals (Houston, TX, USA); and verapamil and cycloheximide from Sigma-Aldrich (St. Louis, MO, USA).

Generation of ABT-199-R cell lines. The DLBCL cell lines, SU-DHL-6 and OCL-LY-19, were made resistant to ABT-199 as previously described. ${ }^{24}$ Briefly, cells were intermittently incubated with a low concentration (fivefold lower than $\mathrm{IC}_{50}$ ) of ABT-199 for short intervals over time and allowed to recover after washing off the drug. The ABT-199 concentration and treatment time were gradually increased until cells remained viable after a continuous exposure to the drug that was double the concentration of their $\mathrm{IC}_{50}$ value. To make sure that cells were not becoming resistant via increased expression of efflux pumps, cells were treated intermittently with verapamil. The ABT199-R cells were routinely tested for resistance to ABT-199 and cultured without drug for $72 \mathrm{~h}$ before they were used in experiments.

Flow cytometry. Cell viability was measured by phosphatidylserine externalization by staining the cells with fluorescein-conjugated Annexin V-PI (BD Biosciences, San Jose, CA, USA). The analysis was done on a BD FACS Calibur flow cytometer (BD Biosciences), and the raw data was processed using CellQuest Version 5.2.1 software (BD Biosciences, Franklin Lakes, NJ, USA). The results were normalized to survival of vehicle control cells treated with ethanol or dimethyl sulfoxide.

Immunoblotting and immunoprecipitation. The cell pellets were lysed with 1\% NP-40 lysis buffer 20 mmol// Tris-HCl, pH 7.5; 1 mmol// EDTA; 150 mmol/l $\mathrm{NaCl} ; 1 \% \mathrm{NP}-40$ ), containing phosphatase inhibitor cocktails 2 and 3 (Sigma) and protease inhibitors (Roche, Indianapolis, IN, USA), for $30-45 \mathrm{~min}$ at $4^{\circ} \mathrm{C}$. Protein lysates were prepared after calculating protein concentration using Bradford reagent (Bio-Rad, Hercules, CA, USA); $50 \mu \mathrm{g}$ of protein was resolved on $10-12 \%$ SDS-PAGE followed by transferring to nitrocellulose or PVDF membranes (Millipore, Danvers, MA, USA). ${ }^{24}$ The immunoblotting was performed with the primary antibodies mentioned below. For immunoprecipitation, protein lysates were prepared by lysing cell pellets with CHAPS buffer $(20 \mathrm{mmol} / \mathrm{l}$ Tris- $\mathrm{HCl}$, pH 7.5; $150 \mathrm{mmol} / / \mathrm{NaCl} ; 1 \mathrm{mmol} / / \mathrm{EDTA} ; 2 \%$ CHAPS; Calbiochem, Billerica, MA, USA) containing protease and phosphatase inhibitors for $1 \mathrm{~h}$ on ice. Protein lysates were incubated with primary antibody overnight at $4{ }^{\circ} \mathrm{C}$, then an equal amount of protein A agarose beads (Calbiochem) was added to all samples, followed by $1 \mathrm{~h}$ of incubation at $4^{\circ} \mathrm{C}$. The beads were washed three times with CHAPS, eluted with loading buffer supplemented with 2-mercaptoethanol (Sigma-Aldrich) and western blotting was performed as mentioned above. BAX activation was determined as described elsewhere. ${ }^{24}$ Primary antibodies used were for MCL-1, BIM, BAX-6A7 (BD Biosciences), NOXA (Enzo Life Sciences, Farmingdale, NY, USA), BCL-2, BCLXL, BAX-N20 (Santa Cruz Biotechnology, Santa Cruz, CA, USA), PUMA (ProSci Incorporated, Poway, CA, USA), cleaved caspase-3, total-AKT, p-AKT (Ser473), p-AKT (Ser308), total-p-70S6 Kinase, p-p70S6 Kinase (Thr389), p-4EBP1 (Ser65) (Cell Signaling Technologies, Danvers, MA, USA), and $\beta$-actin (Sigma). The secondary anti-mouse and -rabbit antibodies were purchased from Thermo-Fisher Scientific (Pittsburg, PA, USA). Protein levels were quantified by ImageJ (NIH, Bethesda, MD, USA); the relative intensity of each lane with respect to control at $0 \mathrm{~h}$ was calculated after normalizing it to the relative intensity of $\beta$-actin.

RNA isolation and real-time quantitative-PCR. RNA was isolated by the Trizol method (Life Technologies) from parental and ABT199-R cells after ABT-737 treatment. Levels of mRNA were analyzed using a quantitative real-time, reverse transcriptase PCR (qRT-PCR) kit (Life Technologies), with primers for Mcl-1, $B C l-x L$, and $B c l-2$, and normalized for $\beta$-actin, as described. ${ }^{15,24}$

siRNA transfection. AKT, MCL-1, and BCL-xL knockdown was achieved using specific siRNA or siControl (Santa Cruz Biotechnology) by Amaxa Nucleofector Kit V (Lonza, Walkersville, MD, USA) (program number 0-007) according to the manufacturer's protocol.

Statistical analysis. Statistical comparisons between groups were conducted by student $t$-test and protein half-life was analyzed using one-phase exponential decay model in Prism (Version 4.0c, GraphPad software Inc, La Jolla, CA, USA). The standard deviation was calculated from experiments conducted in triplicate and is indicated by error bars on the figures. All experiments were repeated three times independently.

\section{Conflict of Interest}

The authors declare no conflict of interest.

Acknowledgements. We would like to thank Drs. Y. Sountharajah, N. Gupta (Cleveland Clinic), and R. Dalla Favera (Columbia University Medical Center) for providing cell lines, and Dr. C. Talerico (Cleveland Clinic) for providing substantive editing and comments. This study was supported by the National Institutes of Health (NCl) grant CA127264 to A. Almasan.

1. Alizadeh AA, Eisen MB, Davis RE, Ma C, Lossos IS, Rosenwald A et al. Distinct types of diffuse large B-cell lymphoma identified by gene expression profiling. Nature 2000; 403: 503-511.

2. Davis RE, Ngo VN, Lenz G, Tolar P, Young RM, Romesser PB et al. Chronic active B-cell-receptor signalling in diffuse large B-cell lymphoma. Nature 2010; 463: 88-92.

3. Rosenwald A, Wright G, Chan WC, Connors JM, Campo E, Fisher RI et al. The use of molecular profiling to predict survival after chemotherapy for diffuse large-B-cell lymphoma. N Engl J Med 2002; 346: 1937-1947. 
4. Fabbri G, Khiabanian H, Holmes AB, Wang J, Messina M, Mullighan CG et al. Genetic lesions associated with chronic lymphocytic leukemia transformation to Richter syndrome. J Exp Med 2013; 210: 2273-2288.

5. Fruman DA, Rommel C. PI3K and cancer: lessons, challenges and opportunities. Nat Rev Drug Discov 2014; 13: 140-156.

6. Iqbal J, Neppalli VT, Wright G, Dave BJ, Horsman DE, Rosenwald A et al. BCL2 expression is a prognostic marker for the activated B-cell-like type of diffuse large B-cell lymphoma. $J$ Clin Oncol 2006; 24: 961-968.

7. Rossi D, Gaidano G. Richter syndrome. Adv Exp Med Biol 2013; 792: 173-191.

8. Vanhaesebroeck B, Khwaja A. PI3Kdelta inhibition hits a sensitive spot in B cell malignancies. Cancer Cell 2014; 25: 269-271.

9. Juin P, Geneste O, Gautier F, Depil S, Campone M. Decoding and unlocking the BCL-2 dependency of cancer cells. Nat Rev Cancer 2013; 13: 455-465.

10. Chipuk JE, Moldoveanu T, Llambi F, Parsons MJ, Green DR. The BCL-2 family reunion. Mol Cell 2010; 37: 299-310.

11. Letai AG. Diagnosing and exploiting cancer's addiction to blocks in apoptosis. Nat Rev Cancer 2008; 8: 121-132

12. van Delft MF, Wei AH, Mason KD, Vandenberg CJ, Chen L, Czabotar PE et al. The BH3 mimetic ABT-737 targets selective Bcl-2 proteins and efficiently induces apoptosis via Bak/Bax if Mcl-1 is neutralized. Cancer Cell 2006; 10: 389-399.

13. Oltersdorf T, Elmore SW, Shoemaker AR, Armstrong RC, Augeri DJ, Belli BA et al. An inhibitor of Bcl-2 family proteins induces regression of solid tumours. Nature 2005; 435 : 677-681.

14. Tse C, Shoemaker AR, Adickes J, Anderson MG, Chen J, Jin S et al. ABT-263: a potent and orally bioavailable Bcl-2 family inhibitor. Cancer Res 2008; 68: 3421-3428.

15. Al-Harbi S, Hill BT, Mazumder S, Singh K, Devecchio J, Choudhary G et al. An antiapoptotic $\mathrm{BCL}-2$ family expression index predicts the response of chronic lymphocytic leukemia to ABT-737. Blood 2011; 118: 3579-3590.

16. Roberts AW, Seymour JF, Brown JR, Wierda WG, Kipps TJ, Khaw SL et al. Substantial susceptibility of chronic lymphocytic leukemia to BCL2 inhibition: results of a phase I study of navitoclax in patients with relapsed or refractory disease. J Clin Oncol 2012; 30: 488-496.

17. Souers AJ, Leverson JD, Boghaert ER, Ackler SL, Catron ND, Chen J et al. ABT-199, a potent and selective BCL-2 inhibitor, achieves antitumor activity while sparing platelets. Nat Med 2013; 19: 202-208.

18. Davids MS, Letai A. ABT-199: taking dead aim at BCL-2. Cancer Cell 2013; 23: 139-141.

19. Pan R, Hogdal LJ, Benito JM, Bucci D, Han L, Borthakur G et al. Selective BCL-2 inhibition by ABT-199 causes on-target cell death in acute myeloid leukemia. Cancer Discov 2014; 4 362-375.

20. Touzeau C, Dousset C, Le Gouill S, Sampath D, Leverson JD, Souers AJ et al. The Bcl-2 specific $\mathrm{BH} 3$ mimetic $\mathrm{ABT}-199$ : a promising targeted therapy for $\mathrm{t}(11 ; 14)$ multiple myeloma. Leukemia 2014; 28: 210-212.

21. Vaillant F, Merino D, Lee L, Breslin K, Pal B, Ritchie ME et al. Targeting BCL-2 with the BH3 mimetic ABT-199 in estrogen receptor-positive breast cancer. Cancer Cell 2013; 24: 120-129.

22. Vandenberg CJ, Cory S. ABT-199, a new Bcl-2-specific BH3 mimetic, has in vivo efficacy against aggressive Myc-driven mouse lymphomas without provoking thrombocytopenia. Blood 2013; 121: 2285-2288.

23. Garber K. Kinase inhibitors overachieve in CLL. Nat Rev Drug Discov 2014; 13: 162-164.

24. Mazumder S, Choudhary GS, Al-Harbi S, Almasan A. Mcl-1 Phosphorylation defines ABT-737 resistance that can be overcome by increased NOXA expression in leukemic B cells. Cancer Res 2012; 72: 3069-3079.

25. Coloff JL, Macintyre AN, Nichols AG, Liu T, Gallo CA, Plas DR et al. Akt-dependent glucose metabolism promotes $\mathrm{Mcl}-1$ synthesis to maintain cell survival and resistance to $\mathrm{Bcl}-2$ inhibition. Cancer Res 2011; 71: 5204-5213.

26. Longo PG, Laurenti L, Gobessi S, Sica S, Leone G, Efremov DG. The Akt/Mcl-1 pathway plays a prominent role in mediating antiapoptotic signals downstream of the $B$-cell receptor in chronic lymphocytic leukemia B cells. Blood 2008; 111: 846-855.

27. Hsieh AC, Costa M, Zollo O, Davis C, Feldman ME, Testa JR et al. Genetic dissection of the oncogenic mTOR pathway reveals druggable addiction to translational control via 4EBPelF4E. Cancer Cell 2010; 17: 249-261.

28. Thomas LW, Lam C, Edwards SW. Mcl-1; the molecular regulation of protein function. FEBS Lett 2010; 584: 2981-2989.
29. Jacquin MA, Chiche J, Zunino B, Beneteau M, Meynet O, Pradelli LA et al. GAPDH binds to active Akt, leading to $\mathrm{Bcl}-\mathrm{xL}$ increase and escape from caspase-independent cell death. Cell Death Differ 2013; 20: 1043-1054.

30. Zang C, Eucker J, Liu H, Muller A, Possinger K, Scholz CW. Concurrent inhibition of PI3kinase and $m T O R$ induces cell death in diffuse large $B$ cell lymphomas, a mechanism involving down regulation of Mcl-1. Cancer Lett 2013; 339: 288-297.

31. Wei MC, Zong WX, Cheng EH, Lindsten T, Panoutsakopoulou V, Ross AJ et al. Proapoptotic BAX and BAK: a requisite gateway to mitochondrial dysfunction and death. Science 2001; 292: 727-730.

32. Bodo J, Zhao X, Sharma A, Hill BT, Portell CA, Lannutti BJ et al. The phosphatidylinositol 3-kinases (PI3K) inhibitor GS-1101 synergistically potentiates histone deacetylase inhibitorinduced proliferation inhibition and apoptosis through the inactivation of PI3K and extracellular signal-regulated kinase pathways. Br J Haematol 2013; 163: 72-80.

33. Sharma A, Janocha TA, Hill BT, Smith MR, Erzurum SC, Almasan A. Targeting mTORC1mediated metabolic addiction to overcome fludarabine resistance in malignant $B$ cells. Mol Cancer Res 2014; 12: 1205-1215.

34. Perciavalle RM, Opferman JT. Delving deeper: MCL-1's contributions to normal and cancer biology. Trends Cell Biol 2013; 23: 22-29.

35. Fresquet V, Rieger M, Carolis C, Garcia-Barchino MJ, Martinez-Climent JA. Acquired mutations in BCL2 family proteins conferring resistance to the $\mathrm{BH} 3$ mimetic ABT-199 in lymphoma. Blood 2014; 123: 4111-4119.

36. Davids MS, Deng J, Wiestner A, Lannutti BJ, Wang L, Wu CJ et al. Decreased mitochondrial apoptotic priming underlies stroma-mediated treatment resistance in chronic lymphocytic leukemia. Blood 2012; 120: 3501-3509.

37. Brown JR, Byrd JC, Coutre SE, Benson DM, Flinn IW, Wagner-Johnston ND et al. Idelalisib, an inhibitor of phosphatidylinositol 3-kinase p110delta, for relapsed/refractory chronic lymphocytic leukemia. Blood 2014; 123: 3390-3397.

38. Fernald K, Kurokawa M. Evading apoptosis in cancer. Trends Cell Biol 2013; 23: 620-633.

39. Rahmani M, Aust MM, Attkisson E, Williams DC Jr., Ferreira-Gonzalez A, Grant S. Dual inhibition of $\mathrm{BCl}-2$ and $\mathrm{Bcl}-\mathrm{xL}$ strikingly enhances $\mathrm{PI}$ KK inhibition-induced apoptosis in human myeloid leukemia cells through a GSK3- and Bim-dependent mechanism. Cancer Res 2013; 73: $1340-1351$.

40. Martelli AM, Evangelisti C, Chappell W, Abrams SL, Basecke J, Stivala F et al. Targeting the translational apparatus to improve leukemia therapy: roles of the PI3K/PTEN/Akt/mTOR pathway. Leukemia 2011; 25: 1064-1079.

41. Billard C. BH3 mimetics: status of the field and new developments. Mol Cancer Ther 2013; 12: $1691-1700$

42. Doi K, Li R, Sung SS, Wu H, Liu Y, Manieri W et al. Discovery of marinopyrrole A (maritoclax) as a selective Mcl-1 antagonist that overcomes ABT-737 resistance by binding to and targeting Mcl-1 for proteasomal degradation. J Biol Chem 2012; 287: 10224-10235.

43. Abulwerdi F, Liao C, Liu M, Azmi AS, Aboukameel A, Mady AS et al. A novel small-molecule inhibitor of mcl-1 blocks pancreatic cancer growth in vitro and in vivo. Molec Cancer Ther 2014; 13: 565-575.

44. Lannutti BJ, Meadows SA, Herman SE, Kashishian A, Steiner B, Johnson AJ et al. CAL-101, a p110delta selective phosphatidylinositol-3-kinase inhibitor for the treatment of B-cell malignancies, inhibits PI3K signaling and cellular viability. Blood 2011; 117: $591-594$.

45. Sharma A, Singh K, Mazumder S, Hill BT, Kalaycio M, Almasan A. BECN1 and BIM interactions with MCL-1 determine fludarabine resistance in leukemic $B$ cells. Cell Death Dis 2013; 4: e628.

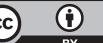

Cell Death and Disease is an open-access journal published by Nature Publishing Group. This work is licensed under a Creative Commons Attribution 4.0 International Licence. The images or other third party material in this article are included in the article's Creative Commons licence, unless indicated otherwise in the credit line; if the material is not included under the Creative Commons licence, users will need to obtain permission from the licence holder to reproduce the material. To view a copy of this licence, visit http://creativecommons.org/licenses/by/4.0 